\title{
Risk Prediction Model Based on Magnetic Resonance Elastography-Assessed Liver Stiffness for Predicting Posthepatectomy Liver Failure in Patients with Hepatocellular Carcinoma
}

Hyo Jung Cho', Young Hwan Ahn'1, Min Suh Sim¹, Jung Woo Eun'1, Soon Sun Kim¹, Bong Wan Kim², Jimi Huh³, Jei Hee Lee ${ }^{3}$, Jai Keun Kim³ ${ }^{3}$ Buil Lee ${ }^{4}$, Jae Youn Cheong ${ }^{3}$, and Bohyun Kim ${ }^{3,5}$

Departments of ${ }^{1}$ Gastroenterology, ${ }^{2}$ Liver Transplantation and Hepatobiliary Surgery, ${ }^{3}$ Radiology, Ajou University School of Medicine, Suwon, ${ }^{4}$ Insight Mining Corporation, Daejeon, and ${ }^{5}$ Department of Radiology, Seoul St. Mary's Hospital, Seoul, Korea

See editorial on page 143.

Article Info

Received March 22, 2021

Revised June 8, 2021

Accepted June 14, 2021

Published online November 23, 2021

Corresponding Author

Jae Youn Cheong

ORCID https://orcid.org/0000-0001-6246-1783

E-mail jaeyoun620@gmail.com

Bohyun Kim

ORCID https://orcid.org/0000-0003-1157-415X

E-mail baboojum@naver.com

Hyo Jung Cho and Young Hwan Ahn contributed equally to this work as first authors.
Background/Aims: Posthepatectomy liver failure (PHLF) is a major complication that increases mortality in patients with hepatocellular carcinoma after surgical resection. The aim of this retrospective study was to evaluate the utility of magnetic resonance elastography-assessed liver stiffness (MRE-LS) for the prediction of PHLF and to develop an MRE-LS-based risk prediction model.

Methods: A total of 160 hepatocellular carcinoma patients who underwent surgical resection with available preoperative MRE-LS data were enrolled. Clinical and laboratory parameters were collected from medical records. Logistic regression analyses were conducted to identify the risk factors for PHLF and develop a risk prediction model.

Results: PHLF was present in 24 patients (15\%). In the multivariate logistic analysis, high MRE-LS (kPa; odds ratio [OR] 1.49, 95\% confidence interval [Cl] 1.12 to 1.98, $\mathrm{p}=0.006$ ), low serum albumin ( $\leq 3.8 \mathrm{~g} / \mathrm{dL}$; OR $15.89,95 \% \mathrm{Cl} 2.41$ to $104.82, \mathrm{p}=0.004$ ), major hepatic resection (OR $4.16,95 \%$ $\mathrm{Cl} 1.40$ to $12.38, \mathrm{p}=0.014)$, higher albumin-bilirubin score (>-0.55; OR $3.72,95 \% \mathrm{Cl} 1.15$ to 12.04 , $\mathrm{p}=0.028$ ), and higher serum $\alpha$-fetoprotein (>100 $\mathrm{ng} / \mathrm{mL}$; OR 3.53, 95\% Cl 1.20 to $10.40, p=0.022$ ) were identified as independent risk factors for PHLF. A risk prediction model for PHLF was established using the multivariate logistic regression equation. The area under the receiver operating characteristic curve (AUC) of the risk prediction model was 0.877 for predicting PHLF and 0.923 for predicting grade $B$ and $C$ PHLF. In leave-one-out cross-validation, the risk model showed good performance, with AUCs of 0.807 for all-grade PHLF and 0. 871 for grade B and C PHLF.

Conclusions: Our novel MRE-LS-based risk model had excellent performance in predicting PHLF, especially grade B and C PHLF. (Gut Liver 2022;16:277-289)

Key Words: Carcinoma, hepatocellular; Hepatectomy, Magnetic resonance elastography; Hepatic fibrosis; Liver failure

\section{INTRODUCTION}

Hepatocellular carcinoma (HCC) is the 6th most common malignancy and is ranked the 4th leading cause of cancer-related mortality worldwide. ${ }^{1-3}$ Surgical resection is the most efficient therapeutic option for selected HCC patients with preserved liver function. ${ }^{4-6}$ With advancements in surgical technique and perioperative management, pos- thepatectomy complications have greatly reduced. However, posthepatectomy liver failure (PHLF) still remains a feared complication as it is a major source of postoperative morbidity and mortality. ${ }^{7-10}$ An accurate risk assessment of PHLF is therefore essential for selecting optimal therapeutic options for HCC patients.

Severity of liver fibrosis is a well-known risk factor of PHLF in HCC patients. ${ }^{11}$ Several ultrasonograms-based 
elastography techniques, including transient elastography (TE) and shear-wave-elastography, have been proposed as non-invasive tools for grading liver fibrosis. Liver stiffness (LS) assessed by TE or shear-wave-elastography has been reported as a significant predictor of PHLF in HCC patients. ${ }^{12-14}$ Magnetic resonance elastography (MRE), an magnetic resonance imaging (MRI)-based tool for the quantitative assessment of LS, is considered the most accurate non-invasive technique for assessing liver fibrosis. ${ }^{15,16}$ Recently, MRE-assessed LS (MRE-LS) has been highlighted as a potential prognostic biomarker in HCC patients. ${ }^{17,18}$ However, little is known about the role of preoperative MRE-LS as a predictor of PHLF in HCC patients.

Clinical risk prediction models for PHLF have been previously developed by many studies, majority of which are based on serum biochemical markers such as albumin, bilirubin, and international normalized ratio. However, diagnostic accuracy of these risk models remains insufficient, with areas under the receiving operating characteristic curve (AUC) ranging between 0.6 and 0.7. ${ }^{19-22}$ Recently, several studies have proposed more comprehensive clinical risk models by combining clinical/biochemical variables with TE-assessed LS for predicting PHLF. ${ }^{23-25}$ Although MRE-LS has demonstrated outstanding performance and reproducibility compared to TE-assessed LS for diagnosing liver fibrosis, no study has derived an MRE-LS-based clinical risk model for predicting PHLF.

In this study, we evaluated the clinical implications of MRE-LS, alongside several clinical parameters for predicting PHLF in HCC patients who underwent surgical resection. Additionally, we developed a comprehensive MRELS-based clinical risk model for assessing the probability of PHLF.

\section{MATERIALS AND METHODS}

\section{Patient selection}

The Institutional Review Board of Ajou Medical Center (IRB number: AJIRB-MED-MDB-18-484) approved this retrospective study and waived the requirement for informed consent. From a prospectively maintained cohort of patients at risk of HCC who underwent MRE, 160 HCC patients who received surgical resection between January 2016 and January 2019 were identified. Of them, 96 patients were included in the prior study exploring the predictive value of MRE-LS for early HCC recurrence after surgical resection. ${ }^{26}$ Fig. 1 illustrates the patient selection process.

\section{LS measurement by MRE}

MRE was performed as part of a routine liver MRI protocol, using 1.5 T (Signa HDxt; GE Healthcare, Chicago, IL, USA) and 3 T scanners (Discovery 750w; GE Healthcare). Hepatic shear wave was generated by $60 \mathrm{~Hz}$ pneumatic vibrations transmitted via an acoustic driver placed on the right upper abdomen of the patient. Motionsensitized 2-dimensional gradient echo and 2-dimensional spin-echo echo-planar MRE sequences were used to visualize hepatic shear waves of the $1.5 \mathrm{~T}$ and $3 \mathrm{~T}$ scanners, respectively. Elastograms were obtained in four contiguous axial images that covered the largest area of right liver.

LS was measured by an abdominal radiologist ( 4 years of experience in liver MRE) on a post-processing software (READY View, version 12.4; GE Healthcare). The largest freehand region of interest (ROI) was manually drawn on non-tumor-bearing areas of the liver parenchyma preferentially in right lobe, bounded by $95 \%$ confidence mapping, while avoiding incoherent shear waves and large vessels on each elastogram. ${ }^{27}$ The ROI was drawn 2 to 3 $\mathrm{cm}$ away from the tumor in reference to the matching axial

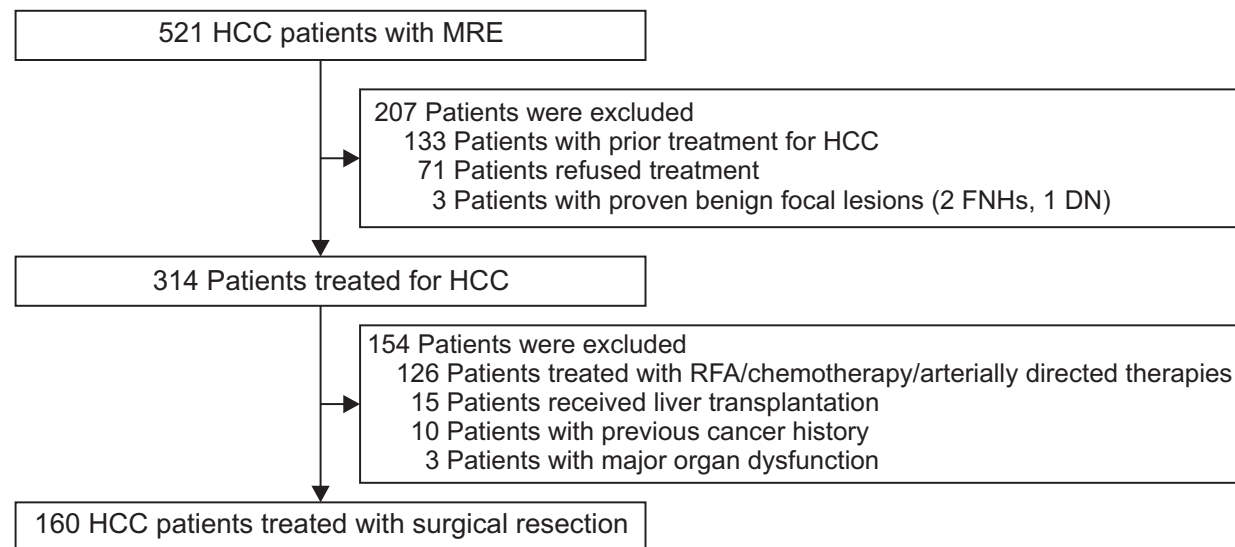

Fig. 1. Flow diagram of subject enrollment.

HCC, hepatocellular carcinoma; MRE, magnetic resonance elastography; FNH, focal nodular hyperplasia; DN, dysplastic nodule; RFA, radiofrequency ablation. 
anatomic images. ${ }^{28}$ LS measured in each image slice (four slice per patient) was averaged to represent LS. The mean size of the ROIs was $31.1 \pm 12.9 \mathrm{~cm}^{2}$.

\section{Treatment of HCC, assessment of outcomes, and definition of terms}

Diagnosis of HCC was based on the American Association for the Study of Liver Diseases practice guidelines, and the European Association for the Study of Liver guidelines. ${ }^{1,29,30}$ Patients underwent hepatic resection with curative intent by one of the two specialized liver surgeons in our institution. Major hepatic resection was defined as surgical removal of $\geq 3$ Couinaud segments. ${ }^{31,32}$

PHLF was evaluated according to the consensus definition and severity grading of the International Study Group of Liver Surgery as follows: PHLF was defined as postoperatively acquired deterioration of liver function, characterized by increased international normalized ratio and concomitant hyperbilirubinemia on or after postoperative day 5. Severity of PHLF was categorized into grades A, B, and C. Grade A PHLF was defined as laboratory confirmation of liver function deterioration requiring no change in clinical management, grade B PHLF requires deviation from regular clinical management, but is manageable without invasive treatment, while grade C PHLF represents a critical clinical condition, resulting in a deviation from regular clinical management and the requirement for invasive treatment. $^{33}$

Resected tumor and non-tumor specimens were examined for the pathological diagnosis of HCC and for the assessment of METAVIR scores by an experienced pathologist (Young-Bae Kim, Department of Pathology, Ajou University School of Medicine with 20 years of experience in liver pathology). The degree of hepatic fibrosis in non-tumor specimens was determined according to the METAVIR score. ${ }^{34}$ Liver disease-specific survival (LSS) was defined as the time from operation day to death from liver disease including liver failure or HCC progression.

Baseline characteristics, including sex, age, etiology, liver cirrhosis on computed tomography or MRI, MRELS, the number of HCC, the number of affected segments, major resection, and laboratory parameters including indocyanine green retention rate at 15 minutes (ICG R15) were retrieved from medical records. Child-Pugh class, Barcelona Clinic Liver Cancer stage, Model for End-Stage Liver Disease (MELD) score, albumin-bilirubin (ALBI) score, and serum fibrosis markers including aspartate aminotransferase-to-platelet ratio (APRI) and fibrosis-4 index (FIB-4) were derived based on medical records. ${ }^{35}$ Functional remnant volume (FRV) was measured for the patients who underwent preoperatively contrast-enhanced computed tomography images of $1 \mathrm{~mm}$ slice. Total liver volume and predicted future remnant liver volume were measured using SYNAPSE VINCENT (Fujifilm Medical, Tokyo, Japan) preoperatively. FRV was calculated as following equation: FRV (\%)=predicted future remnant liver volume/total liver volume $\times 100 \%$.

\section{Statistics}

Statistical analyses were performed using the SPSS soft-

Table 1. Baseline Characteristics and Treatment Outcomes of All Included Patients

\begin{tabular}{|c|c|}
\hline Variable & All patients ( $n=160$ ) \\
\hline Age, yr & $57.1 \pm 9.9$ \\
\hline Male sex & 124 (77.5) \\
\hline \multicolumn{2}{|l|}{ Child-Pugh class } \\
\hline$A$ & $157(98.1)$ \\
\hline $\mathrm{B}$ & $3(1.9)$ \\
\hline MELD & $7.9 \pm 1.7$ \\
\hline Platelet, $\times 10^{9} / \mathrm{L}$ & $170.8 \pm 71.1$ \\
\hline Albumin, g/L & $4.5 \pm 0.4$ \\
\hline Bilirubin, mg/dL & $0.6 \pm 0.3$ \\
\hline AFP, ng/mL & $2,261.90 \pm 9,387.44$ \\
\hline$A L T, U / L$ & $50.5 \pm 96.3$ \\
\hline INR & $1.1 \pm 0.1$ \\
\hline \multicolumn{2}{|l|}{ Underlying liver disease } \\
\hline $\mathrm{CHB}$ & 131 (81.9) \\
\hline $\mathrm{CHC}$ & $9(5.6)$ \\
\hline Alcoholics & $5(3.1)$ \\
\hline Cryptogenic & $15(9.4)$ \\
\hline Tumor size, $\mathrm{cm}$ & $3.8 \pm 2.9$ \\
\hline \multicolumn{2}{|l|}{ BCLC stage } \\
\hline 0 & $37(23.1)$ \\
\hline$A$ & 54 (33.8) \\
\hline $\mathrm{B}$ & $12(7.5)$ \\
\hline $\mathrm{C}$ & $57(35.6)$ \\
\hline $\mathrm{D}$ & 0 \\
\hline \multicolumn{2}{|c|}{ Posthepatectomy liver failure } \\
\hline Grade A & $5(3.1)$ \\
\hline Grade B & $17(10.7)$ \\
\hline Grade C & $2(1.2)$ \\
\hline ICG R15, \% & $14.75 \pm 7.86$ \\
\hline APRI & $0.84 \pm 1.11$ \\
\hline FIB-4 & $2.60 \pm 1.95$ \\
\hline \multicolumn{2}{|c|}{ Pathologic hepatic fibrosis stage } \\
\hline FO & $11(6.9)$ \\
\hline F1 & $4(2.5)$ \\
\hline F2 & $5(3.1)$ \\
\hline F3 & $71(44.4)$ \\
\hline F4 & $69(43.1)$ \\
\hline
\end{tabular}

Data are presented as the mean \pm SD or number $(\%)$.

MELD, Model for End-Stage Liver Disease; AFP, a-fetoprotein; ALT, alanine transaminase; INR, international normalized ratio; $\mathrm{CHB}$, chronic hepatitis $\mathrm{B} ; \mathrm{CHC}$, chronic hepatitis $\mathrm{C} ; \mathrm{BCLC}$, Barcelona Clinic Liver Cancer; ICG R15, indocyanine green retention rate at $15 \mathrm{~min}$ utes; APRI, aspartate aminotransferase-to-platelet ratio index; FIB-4, fibrosis-4. 
ware version 25 (IBM Corp., Armonk, NY, USA) and R statistical software version 4.0.3 (R Foundation for Statistical Computing, Vienna, Austria). Diagnostic accuracy of MRE-LS and serum fibrosis markers for hepatic fibrosis staging was assessed using AUC, and were compared using the DeLong method. For survival analysis, the KaplanMeier analysis and the Cox regression analysis were performed. $\mathrm{p}<0.05$ were considered significant. A logistic regression analysis was performed to identify significant predictors of PHLF. The risk prediction model for PHLF was formulated based on results of the multivariate logistic analysis. Performance of the risk scoring model was evaluated by AUC which were computed by using leave-one-out cross-validation with random forest.

\section{RESULTS}

\section{Baseline characteristics of study subjects}

Table 1 shows the baseline characteristics of all patients. The mean age of the cohort was 57.1 \pm 9.9 years, and 124 patients $(77.5 \%)$ were male. PHLF developed in 24 patients, with five, 17 , and two being grade $\mathrm{A}, \mathrm{B}$, and C PHLF, respectively. In terms of Clavien-Dindo surgical complication classification, ${ }^{36}$ patients with PHLF were classified as following; four patients were grade I, 14 patients were grade II, four patients were grade IIIa, and two patients were grade $\mathrm{V}$.

In terms of tumor burden, the mean tumor size was $3.8 \pm 2.9 \mathrm{~cm}$. While 136 patients $(85 \%)$ had a single tumor, 24 (15\%) had multiple tumors. According to Barcelona Clinic Liver Cancer staging, 37 (23.1\%), 54 (33.8\%), 12 (7.5\%), and 57 (35.6\%) patients were categorized into stage $0, A, B$, and $C$, respectively. Major hepatectomy was performed in 54 patients $(33.8 \%)$. In preoperative imaging modalities including computed tomography or MR, 101 patients $(63.1 \%)$ were diagnosed with liver cirrhosis by the radiologists. The pathological hepatic fibrosis grade was F0 in 11 patients $(7.5 \%), \mathrm{F} 1$ in four $(2.5 \%), \mathrm{F} 2$ in five $(3.1 \%)$, F3 in 71 (44.4\%), and F4 in 69 (43.1\%). Mean preoperative MRE-LS was $4.02 \pm 1.61 \mathrm{kPa}$.

\section{Diagnostic accuracy of MRE-LS and serum biomarkers for assessing pathological hepatic fibrosis stage}

Diagnostic accuracy of MRE-LS and serum fibrosis
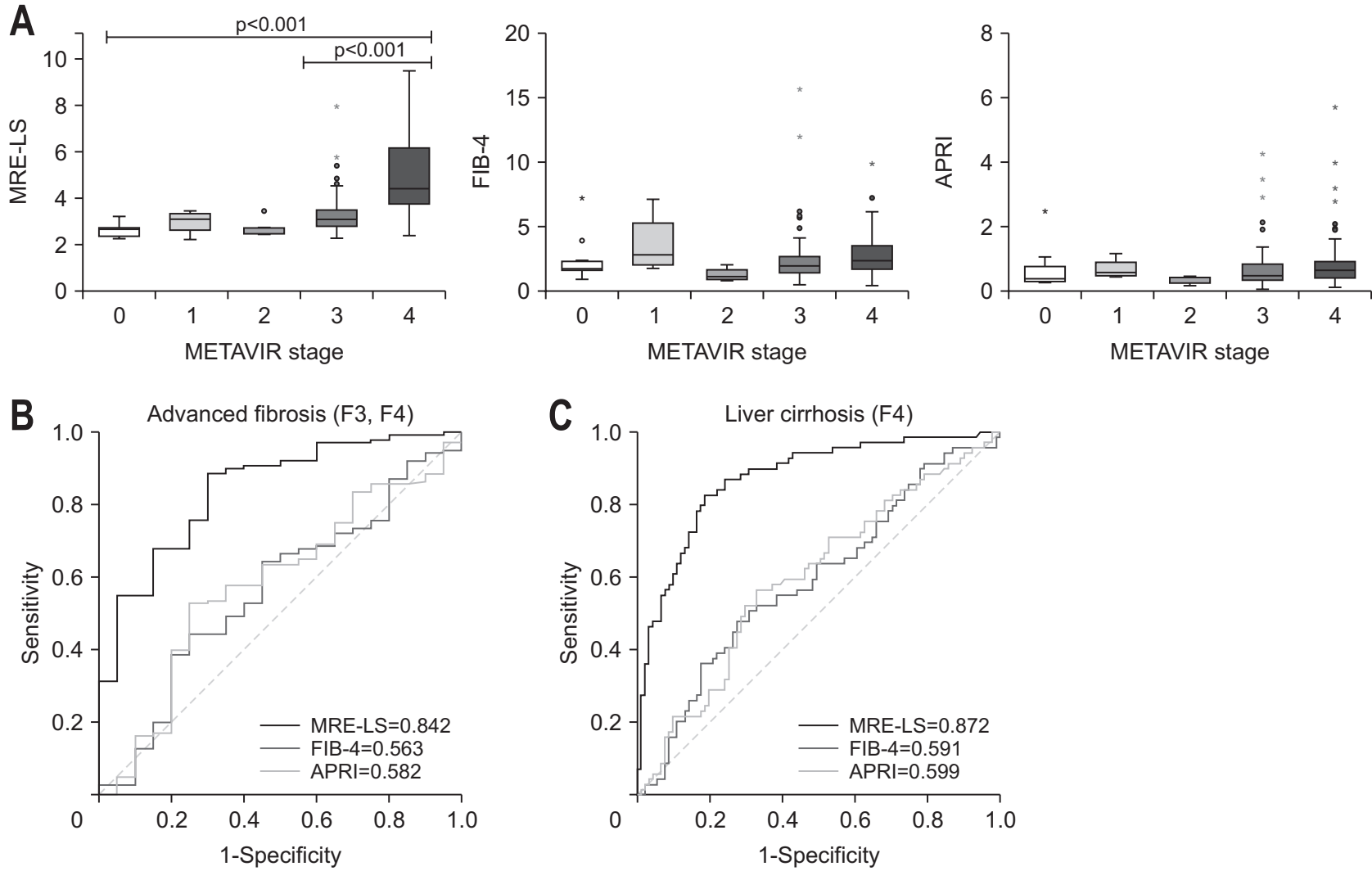

Fig. 2. Diagnostic accuracy of MRE-LS, FIB-4, and APRI for assessing hepatic fibrosis. (A) Value of MRE-LS, FIB-4, and APRI according to METAVIR stage. (B) Comparison of the diagnostic accuracy of MRE-LS, FIB-4, and APRI for assessing advanced fibrosis (F3 or F4). (C) Comparison of the diagnostic accuracy of MRE-LS, FIB-4, and APRI for assessing cirrhosis (F4).

MRE-LS, magnetic resonance elastography-assessed liver stiffness; FIB-4, fibrosis-4; APRI, aspartate aminotransferase-to-platelet ratio index. 
markers for assessing pathological hepatic fibrosis was analyzed (Fig. 2). MRE-LS was observed to gradually increase with METAVIR stage, with statistical significance, while serum fibrosis markers did not show any significant differences (Fig. 2A). For predicting advanced fibrosis $(\geq \mathrm{F} 3)$, MRE-LS showed significantly higher AUC (0.842; 95\% confidence interval [CI], 0.753 to 0.931 ) compared to other serum fibrosis markers $(\mathrm{p}<0.001)$ (Fig. 2B). Likewise, for predicting liver cirrhosis (F4), MRE-LS demonstrated outstanding diagnostic accuracy (AUC, 0.872; 95\% CI, 0.816 to 0.928$)$ compared to others $(\mathrm{p}<0.001)$. The best cutoff MRE-LS value for diagnosing F4 was $3.54 \mathrm{kPa}$, with $82.6 \%$ sensitivity and $81.3 \%$ specificity.

\section{Risk factors for predicting poor survival in HCC patients who underwent surgical resection}

Survival analyses were performed using mortality data obtained from retrospective chart review and the Central Cancer Registry database of the National Cancer Center of South Korea. The median follow-up duration was 944 days, with a range of 37 to 1,572 days. During this period, $14(8.8 \%)$ patients died from underlying liver diseases. Among these 14 mortalities, while 136 patients without PHLF had six mortalities (mortality rate: 4.4\%), 24 patients who developed PHLF demonstrated eight mortalities (mortality rate: $33.3 \%$ ). Among the eight mortalities, only one had grade A PHLF, while seven had grade B or C PHLF. Fig. 3A shows the comparison of LSS between patients with or without PHLF. Patients with PHLF showed poor LLS than patients without PHLF $(\mathrm{p}<0.01)$.

Fig. 3B-E show the comparison of the Kaplan-Meier plots according to selected preoperative parameters. High MRE-LS (>3.8 kPa), high serum $\alpha$-fetoprotein (AFP) level $(>100 \mathrm{ng} / \mathrm{mL})$, low serum albumin level $(\leq 3.8 \mathrm{~g} / \mathrm{dL})$, and major hepatic resection were significantly associated with poor LSS, while preoperative ALBI score, MELD score, ICG R15, and international normalized ratio were not significantly associated with LSS (Supplementary Fig. 1C-F). Supplementary Fig. 1A and B show the Kaplan-Meier plots of LSS according to pathological liver fibrosis stage. No significant differences in LSS were shown between the groups with or without advanced liver fibrosis $(\geq F 3)$, and between those with or without liver cirrhosis (F4).

Cox regression analyses were performed to determine independent risk factors of poor LSS (Table 2). In the univariate analysis, high MRE-LS, low serum albumin level, high MELD score, high serum AFP level, and major hepatic resection were significantly associated with poor LLS. These variables were subsequently entered into the multivariate Cox regression analysis using the backward stepwise selection method. Eventually, high MRE-LS (kPa; hazard ratio, 1.33 ; 95\% $\mathrm{CI}, 1.05$ to $1.69 ; \mathrm{p}=0.018)$, high serum AFP level (>100 ng/mL; hazard ratio, 2.96; 95\% CI, 1.01 to $8.62 ; \mathrm{p}=0.047$ ), and major hepatic resection (hazard ratio, $3.01 ; 95 \% \mathrm{CI}, 1.11$ to $9.82 ; \mathrm{p}=0.031$ ) were identified as independent risk factors for predicting poor LSS in HCC patients who underwent hepatic resection.

\section{Risk factors associated with development of PHLF}

As demonstrated in Fig. 3A, development of PHLF was strongly associated with poor LSS in patients with HCC. Preoperative risk factors associated with PHLF were therefore investigated (Table 3 ). The variables demonstrating significance in univariate analyses were entered into the multivariate analysis using the backward stepwise selection method. High MRE-LS (kPa; odds ratio [OR], 1.49; 95\% CI, 1.12 to $1.98 ; \mathrm{p}=0.006)$, low serum albumin level ( $\leq 3.8 \mathrm{~g} / \mathrm{dL}$; OR, 15.89; 95\% CI, 2.41 to $104.82 ; \mathrm{p}=0.004)$, major hepatic resection (OR, 4.16; $95 \% \mathrm{CI}, 1.40$ to 12.38 ; $\mathrm{p}=0.010)$, high ALBI score $(>-0.55 ; \mathrm{OR}, 3.72 ; 95 \% \mathrm{CI}, 1.15$ to $12.04 ; \mathrm{p}=0.028$ ), and high serum AFP level (>100 ng/ $\mathrm{mL}$; OR, 3.53; 95\% CI, 1.12 to $10.40 ; \mathrm{p}=0.022$ ) were identified as independent risk factors of PHLF.

We performed subgroup analysis in the 100 patients with available FRV to identify clinical implication of FRV in patients who underwent hepatic resection. As a result, FRV was not significantly associated with development of PHLF, whereas MRE-LS demonstrated significant result also in this subgroup (Supplementary Table 1). In the survival analysis, patients with lower FRV (FRV $\leq 40)$ demonstrated significantly poor prognosis $(\mathrm{p}=0.041)$ (Supplementary Fig. 2). In the Cox regression analysis, high MRE-LS was identified as an independent risk factor for predicting poor LSS, whereas FRV was not significant in multivariate analysis (Supplementary Table 2).

\section{Risk model for PHLF}

A risk model for predicting PHLF prior to hepatic resection was developed using the multivariate logistic model as demonstrated in Table 3. By using the $\beta$-coefficients of the multivariate model, the logistic equation for predicting PHLF was derived as follows, and such a model was named the "Comprehensive Risk Model for PHLF (CRMP) index":

$\mathrm{CRMP}$ index $=\left(-5.55+1.4 \mathrm{X}_{1}+1.313 \mathrm{X}_{2}+2.766 \mathrm{X}_{3}+1.426 \mathrm{X}_{4}\right.$ $+1.26 \mathrm{X}_{5}$ ),

where $\mathrm{X}_{1}$ is MRE-LS; $\mathrm{X}_{2}$ is 0 (if $\mathrm{ALBI} \leq-0.55$ ) or $\mathrm{X}_{2}$ is 1 (if ALBI >-0.55); $\mathrm{X}_{3}$ is 1 (if albumin $\leq 3.8 \mathrm{~g} / \mathrm{dL}$ ) or $\mathrm{X}_{3}$ is 0 (if albumin $>3.8 \mathrm{~g} / \mathrm{dL}$ ); $\mathrm{X}_{4}$ is 1 (if major resection) or $\mathrm{X}_{4}$ is 0 (if minor resection); $X_{5}$ is 1 (if $A F P>100 \mathrm{ng} / \mathrm{mL}$ ) or $X_{5}$ is 0 (if AFP $\leq 100 \mathrm{ng} / \mathrm{mL}$ ). 

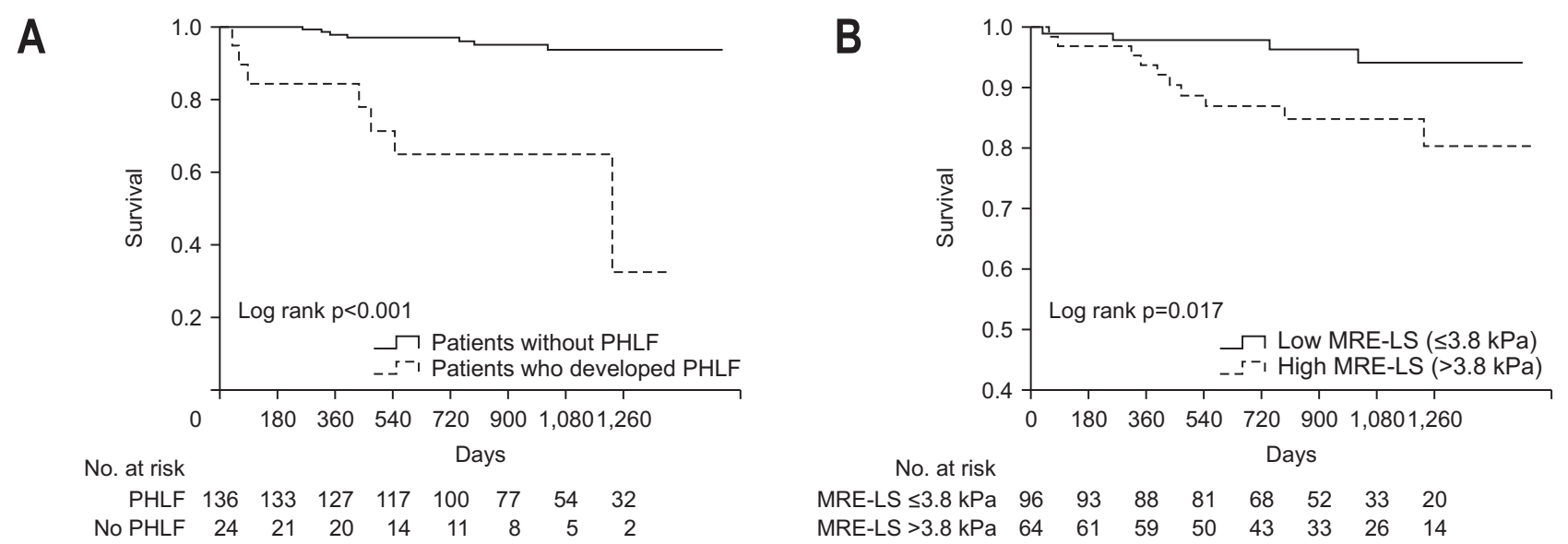

C

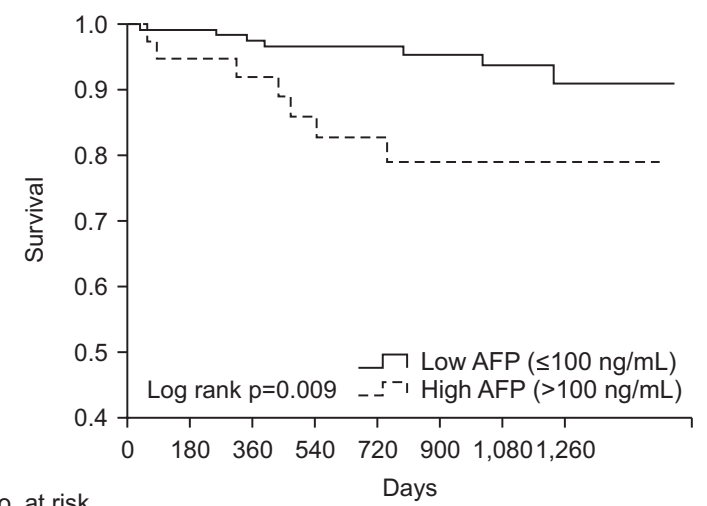

No. at risk

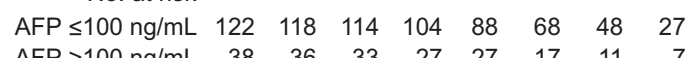

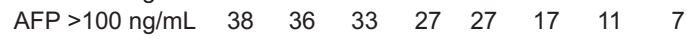

E

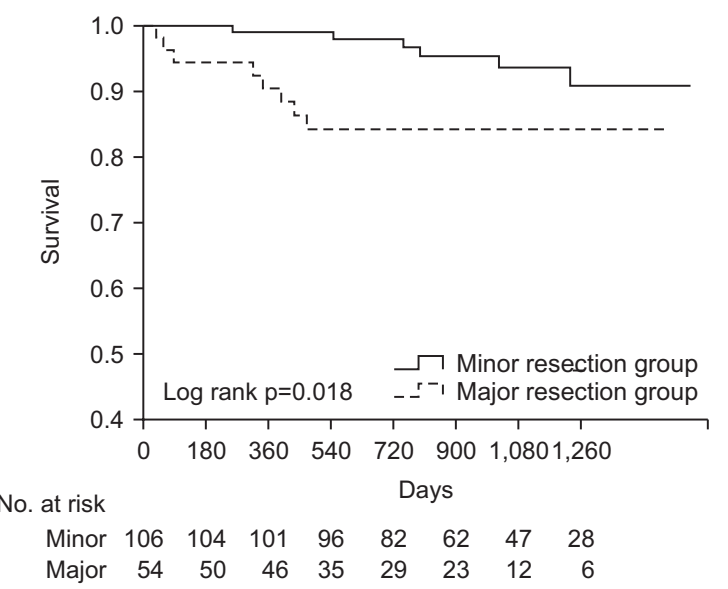

D

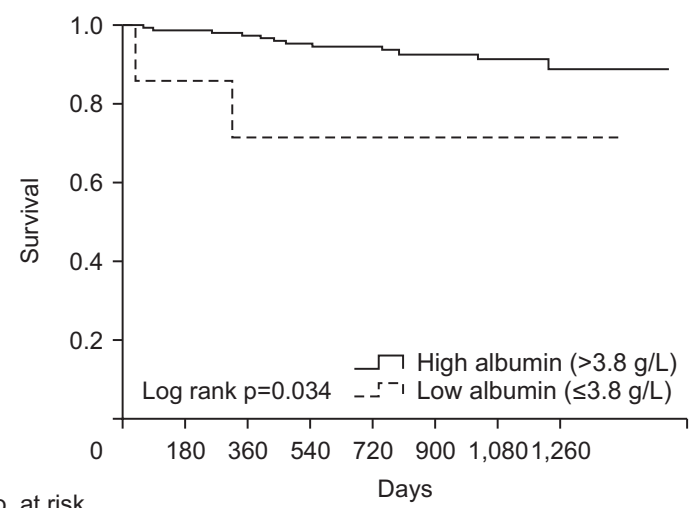

No. at risk

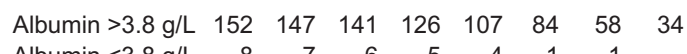

$\begin{array}{llllllll}\text { Albumin }<3.8 \mathrm{~g} / \mathrm{L} & 8 & 7 & 6 & 5 & 4 & 1 & 1\end{array}$

$\mathbf{F}$

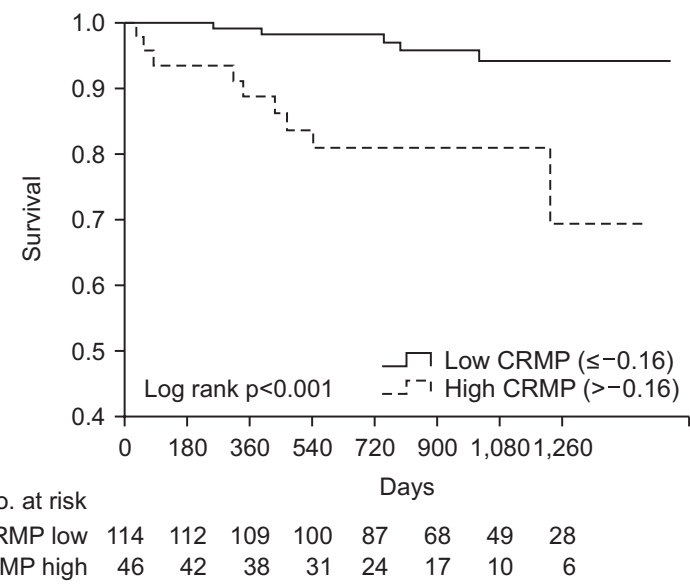

Fig. 3. Kaplan-Meier plots for comparing liver disease-specific survival. (A) Comparison of liver disease-specific survival according to the development of posthepatectomy liver failure (PHLF). (B) Comparison of liver disease-specific survival according to magnetic resonance elastographyassessed liver stiffness (MRE-LS). (C) Comparison of liver disease-specific survival according to serum a-fetoprotein (AFP). (D) Comparison of liver disease-specific survival according to serum albumin. (E) Comparison of liver disease-specific survival according to the development of operation method. (F) Comparison of liver disease-specific survival according to comprehensive risk model for PHLF (CRMP) index. 
Cho HJ, et al: Comprehensive Risk Model for PHLF-Based on MR Elastography

Table 2. Univariate and Multivariate Analyses for Identifying Risk Factors for Poor Liver Disease-Specific Survival in All Included Patients

\begin{tabular}{|c|c|c|c|c|}
\hline \multirow{2}{*}{ Factor } & \multicolumn{2}{|c|}{ Univariate analysis } & \multicolumn{2}{|c|}{ Multivariate analysis } \\
\hline & $\mathrm{HR}(95 \% \mathrm{Cl})$ & p-value & $\mathrm{HR}(95 \% \mathrm{Cl})$ & $p$-value \\
\hline Male sex & $0.67(0.24-1.91)$ & 0.455 & & \\
\hline Age & 0.98 (0.93-1.03) & 0.460 & & \\
\hline Cirrhosis in $\mathrm{CT}$ or MR & $1.48(0.46-4.72)$ & 0.509 & & \\
\hline MRE-LS & $1.32(1.05-1.68)$ & $0.020^{*}$ & $1.33(1.05-1.69)$ & $0.018^{*}$ \\
\hline Albumin & $0.34(0.12-0.94)$ & $0.038^{*}$ & & \\
\hline Total bilirubin & $1.37(0.29-6.50)$ & 0.693 & & \\
\hline INR & $10.75(0.37-310.69)$ & 0.166 & & \\
\hline Platelet & $1.00(0.99-1.01)$ & 0.669 & & \\
\hline BCLC stage, B or C & $2.54(0.85-7.57)$ & 0.096 & & \\
\hline Major resection, yes & $3.37(1.16-9.79)$ & $0.025^{*}$ & $3.01(1.11-9.82)$ & $0.031^{*}$ \\
\hline MELD & $1.23(1.01-1.51)$ & $0.038^{*}$ & & \\
\hline ICG R15 & $1.02(0.96-1.08)$ & 0.615 & & \\
\hline ALBI & $1.65(0.05-53.15)$ & 0.777 & & \\
\hline AFP $>100$ ng/mL & 3.69 (1.29-10.53) & $0.015^{*}$ & $2.96(1.01-8.62)$ & $0.047^{*}$ \\
\hline APRI & $1.01(0.61-1.67)$ & 0.980 & & \\
\hline FIB-4 & $1.07(0.87-1.32)$ & 0.523 & & \\
\hline
\end{tabular}

$\mathrm{HR}$, hazard ratio; $\mathrm{Cl}$, confidence interval; $\mathrm{CT}$, computed tomography; MR, magnetic resonance; MRE-LS, magnetic resonance elastographyassessed liver stiffness; INR, international normalized ratio; BCLC, Barcelona Clinic Liver Cancer; MELD, Model for End-Stage Liver Disease; ICG $\mathrm{R} 15$, indocyanine green retention rate at 15 minutes; ALBI, albumin-bilirubin; AFP, a-fetoprotein; APRI, aspartate aminotransferase-to-platelet ratio index; FIB-4, fibrosis-4.

*Statistically significant, $p<0.05$.

Table 3. Univariate and Multivariate Analyses for Risk Factors for Posthepatectomy Liver Failure

\begin{tabular}{|c|c|c|c|c|c|}
\hline \multirow{2}{*}{ Factor } & \multicolumn{2}{|c|}{ Univariate analysis } & \multicolumn{2}{|c|}{ Multivariate analysis } & \multirow{2}{*}{ Coefficient } \\
\hline & OR* $(95 \% \mathrm{CI})$ & p-value & OR $(95 \% \mathrm{Cl})$ & $p$-value & \\
\hline Male sex & $1.18(0.43-3.23)$ & 0.751 & & & \\
\hline Age & $0.99(0.95-1.03)$ & 0.619 & & & \\
\hline Cirrhosis in CT or MR & $0.97(0.40-2.38)$ & 0.945 & & & \\
\hline MRE-LS & $1.52(1.20-1.92)$ & $0.001 *$ & $1.49(1.12-1.98)$ & $0.006^{*}$ & 0.400 \\
\hline Albumin $\leq 3.8 \mathrm{~g} / \mathrm{dL}$ & $11.67(2.58-52.81)$ & $0.001 *$ & 15.89 (2.41-104.82) & $0.004^{*}$ & 2.766 \\
\hline Total bilirubin $\geq 1.0 \mathrm{mg} / \mathrm{dL}$ & $3.69(0.99-13.74)$ & 0.091 & & & \\
\hline INR & $19.96(0.56-712.06)$ & 0.101 & & & \\
\hline Platelet & $1.00(0.99-1.01)$ & 0.593 & & & \\
\hline Major resection, yes & $3.36(1.38-8.19)$ & $0.008^{*}$ & $4.16(1.40-12.38)$ & $0.010^{*}$ & 1.426 \\
\hline MELD & $1.24(1.00-1.54)$ & $0.048^{*}$ & & & \\
\hline ICG R15 & $1.02(0.97-1.07)$ & 0.393 & & & \\
\hline ALBI >- 0.55 & $3.17(1.23-8.14)$ & $0.017^{*}$ & $3.72(1.15-12.04)$ & $0.028 *$ & 1.313 \\
\hline AFP $>100 \mathrm{ng} / \mathrm{mL}$ & $3.42(1.38-8.46)$ & $0.008^{*}$ & $3.53(1.20-10.40)$ & $0.022^{*}$ & 1.260 \\
\hline APRI & $1.07(0.76-1.52)$ & 0.706 & & & \\
\hline FIB-4 & $1.07(0.88-1.30)$ & 0.481 & & & \\
\hline BCLC stage, $\mathrm{B}$ or $\mathrm{C}$ & $1.69(0.71-4.04)$ & 0.239 & & & \\
\hline Child-Pugh class, B & $2.91(0.25-33.45)$ & 0.391 & & & \\
\hline
\end{tabular}

OR, odds ratio; $\mathrm{Cl}$, confidence interval; $\mathrm{CT}$, computed tomography; MR, magnetic resonance; MRE-LS, magnetic resonance elastography-assessed liver stiffness; INR, international normalized ratio; MELD, Model for End-Stage Liver Disease; ICG R15, indocyanine green retention rate at 15 minutes; ALBI, albumin-bilirubin; AFP, a-fetoprotein; APRI, aspartate aminotransferase-to-platelet ratio index; FIB-4, fibrosis-4; BCLC, Barcelona Clinic Liver Cancer.

*Statistically significant, $p<0.05$.

\section{CRMP index: discriminative performance for predicting PHLF and prognostic role for predicting LSS in all included patients}

Fig. $4 \mathrm{~A}$ shows the receiving operating characteristic curves of the CRMP index and of other potential biomarkers for assessing PHLF. The CRMP index demonstrated the highest predictive power for PHLF compared with single biomarkers (AUC, 0.877; 95\% CI, 0.805 to 0.948 ), 
A

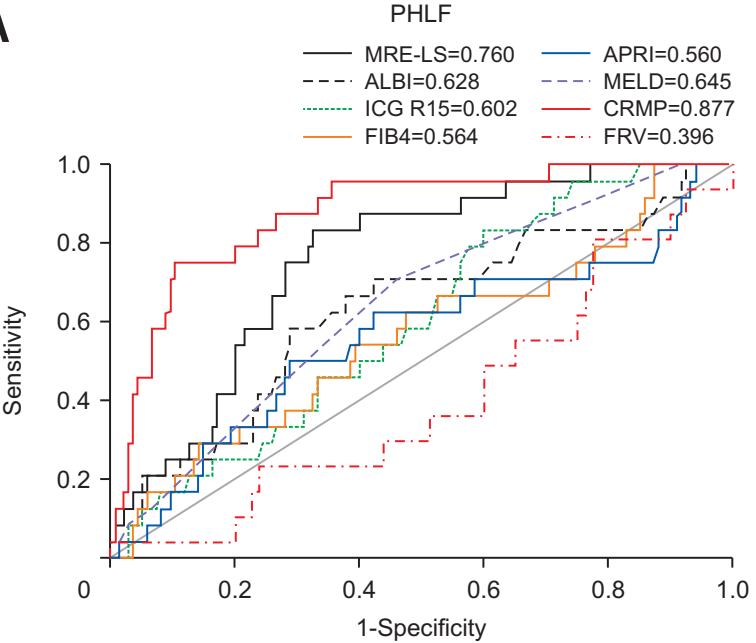

C
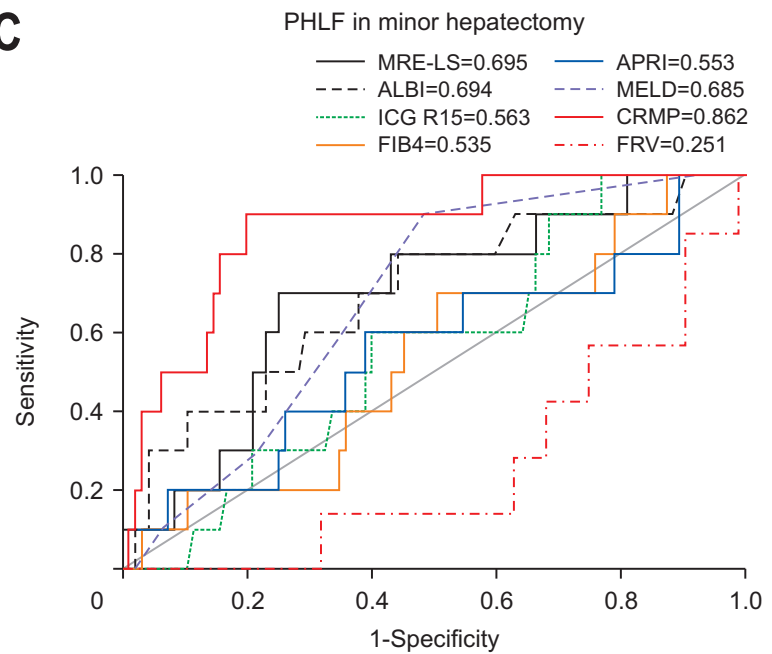

E
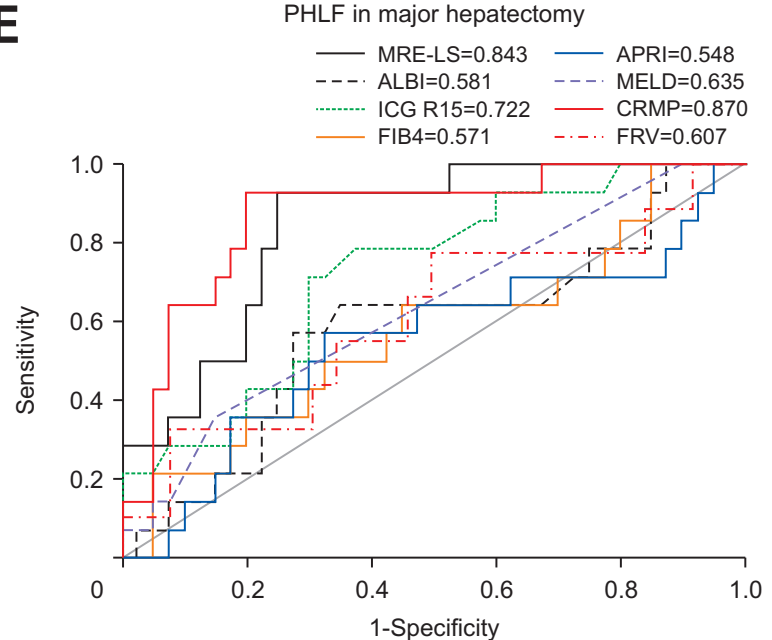

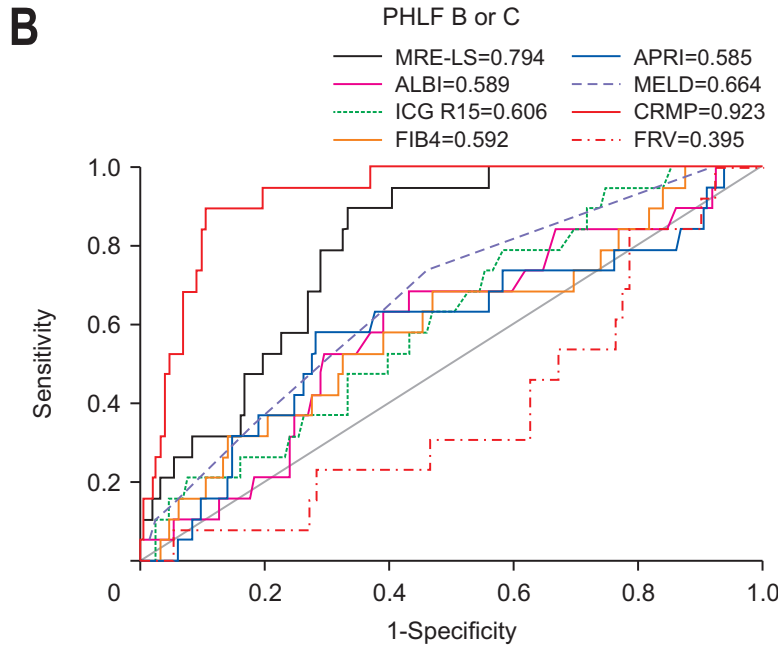

D

PHLF B or C in minor hepatectomy
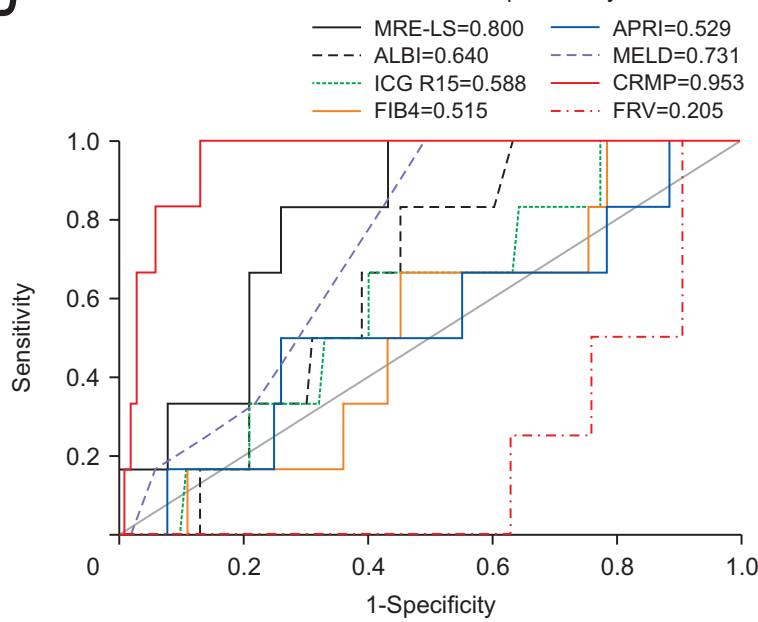

$\mathbf{F}$

PHLF B or C in major hepatectomy
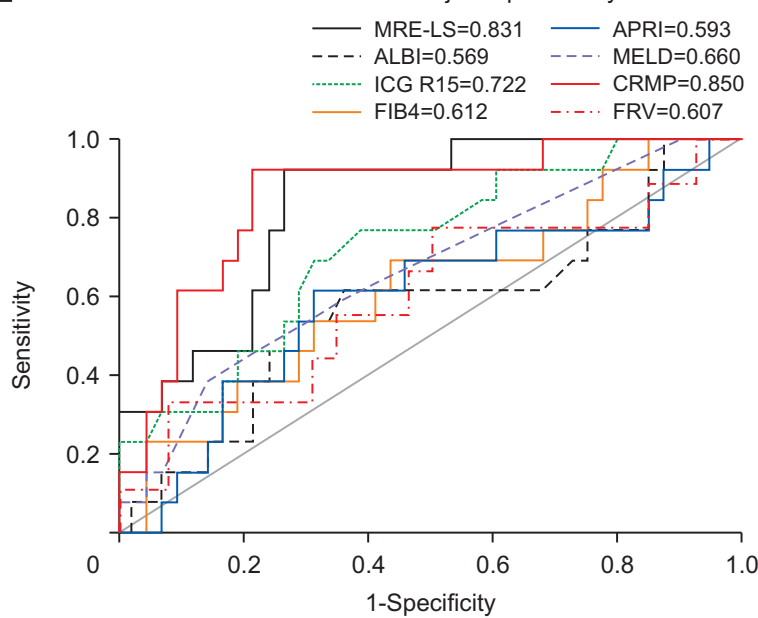

Fig. 4. Areas under the curve of MRE-LS, ALBI score, ICG R15, FIB-4, APRI, MELD, CRMP, and FRV index for assessing posthepatectomy liver failure (PHLF). Areas under the curve for assessing the development of PHLF (A) and grade B or C PHLF (B) in all included patients. Areas under the curve for assessing the development of PHLF (C) and grade B or C PHLF (D) in the minor hepatectomy group. Areas under the curve for assessing the development of PHLF (E) and grade B or C PHLF (F) in the major hepatectomy group.

MRE-LS, magnetic resonance elastography-assessed liver stiffness; ALBI, albumin-bilirubin; ICG R15, indocyanine green retention rate at 15 minutes; FIB-4, fibrosis-4; APRI, aspartate aminotransferase-to-platelet ratio index; MELD, Model for End-Stage Liver Disease; CRMP, comprehensive risk model for PHLF; FRV, functional reserve volume. 
Table 4. Validation of CRMP Index Using Leave-One-Out Cross-Validation with Random Forest

\begin{tabular}{lcc}
\hline \multicolumn{1}{c}{ Variable } & $\begin{array}{c}\text { For predicting } \\
\text { PHLF }\end{array}$ & $\begin{array}{c}\text { For predicting } \\
\text { grade B or C PHLF }\end{array}$ \\
\hline Accuracy & 0.839 & 0.871 \\
AUC & 0.801 & 0.929 \\
Sensitivity, \% & 85.2 & 92.9 \\
Specificity, \% & 75.0 & 33.3 \\
Positive predictive value, \% & 95.8 & 92.9 \\
Negative predictive value, \% & 42.9 & 33.3 \\
\hline
\end{tabular}

PHLF, posthepatectomy liver failure; CRMP, comprehensive risk model for PHLF; AUC, area under the receiving operating characteristic curve.

followed by MRE-LS (AUC, $0.760 ; 95 \%$ CI, 0.669 to 0.851 ), ALBI score (AUC, $0.628 ; 95 \%$ CI, 0.501 to 0.754 ), ICG R15 (AUC, 0.602; 95\% CI, 0.493 to 0.711), FIB-4 (AUC, 0.564; 95\% CI, 0.434 to 0.694 ), and APRI (AUC, 0.560; 95\% CI, 0.424 to 0.696 ). The best cutoff value for the CRMP index in predicting PHLF was -1.9 , with $87.5 \%$ sensitivity and $73.3 \%$ specificity. Statistical comparison of the AUCs between the biomarkers is demonstrated in Supplementary Table 3. Leave-one-out cross-validation (LOOCV) was performed to estimate the performance of CMRP index. Table 4 shows performance of CRMP index in LOOCV. CRMP index showed relatively good performance (AUC, 0.801; accuracy, 0.839) in LOOCV.

Likewise, for predicting grade B and C PHLF, the CRMP index showed outstanding performance (AUC, 0.923; 95\% CI, 0.874 to 0.971 ) (Fig. $4 \mathrm{~A}$ and B). The best cutoff value for the CRMP index in predicting grade $\mathrm{B}$ and C PHLF was -1.6 , with $94.7 \%$ sensitivity and $80.0 \%$ specificity. MRE-LS was the second most potent biomarker for predicting grade B and C PHLF (AUC, 0.794; 95\% CI, 0.713 to 0.876 ), with the best cutoff value being $3.8 \mathrm{kPa}$, with $89.5 \%$ sensitivity and $66.4 \%$ specificity. In LOOCV, AUC of CRMP index for predicting grade B and C PHLF was calculated as 0.929 (accuracy, 0.871) (Table 4).

Fig. 3F shows the Kaplan-Meier plot of LSS according to the CRMP index. Patients with a high CRMP index demonstrated markedly poorer prognosis than those with a low CRMP index $(\mathrm{p}<0.001)$.

\section{AUCs of biomarkers for predicting PHLF in patients who underwent minor hepatic resection}

In the subgroup of patients who underwent minor hepatic resection, AUC of the CRMP index was $0.862(95 \%$ CI, 0.753 to 0.971 ) and that of MRE-LS was 0.695 (95\% CI, 0.533 to 0.857 ) (Fig. $4 \mathrm{C}$ and D). While AUC of the CRMP index was significantly higher than that of ICG R15, FIB4, APRI, ALBI score, and MELD score, no significant differences between AUCs of the CRMP index and MRE-LS were observed in this subgroup ( $\mathrm{p}=0.085)$ (Supplementary Table 3).

For predicting grade $\mathrm{B}$ and $\mathrm{C}$ PHLF in the minor resection subgroup, AUC of the CRMP index showed outstanding performance (AUC, $0.953 ; 95 \% \mathrm{CI}, 0.907$ to 0.999 ), followed by MRE-LS (AUC, $0.800 ; 95 \%$ CI, 0.674 to 0.925 ), ALBI score (AUC, 0.640; 95\% CI, 0.493 to 0.786), ICG R15 (AUC, 0.529), APRI (AUC, 0.529), and FIB-4 (AUC, 0.515) (Fig. 4C and D). The CRMP index showed significantly higher AUC than other single biomarkers (Supplementary Table 3). The best cutoff value for the CRMP index in predicting grade $\mathrm{B}$ and $\mathrm{C}$ PHLF in the minor resection subgroup was 0.17 , with $100 \%$ sensitivity and $87 \%$ specificity.

\section{AUCs for predicting PHLF in patients who underwent major hepatic resection}

In the subgroup underwent major hepatic resection, AUC of the CRMP index was 0.870 (95\% CI, 0.761 to 0.978), while that of MRE-LS was 0.843 (95\% CI, 0.736 to 0.949 ) (Fig. $4 \mathrm{E}$ and F). No significant differences between AUCs of MRE-LS and the CRMP index were shown $(\mathrm{p}=0.687)$. AUCs of MRE-LS and the CRMP index were, however, significantly higher than those of other biomarkers (Supplementary Table 3). AUCs of ICG R15, ALBI score, FIB-4, and APRI were 0.722, 0.581, 0.571, and 0.548, respectively. In the major resection subgroup, the best cutoff value for the CRMP index in predicting PHLF was 0.22 , with $92.9 \%$ sensitivity and $80 \%$ specificity.

In predicting grade $\mathrm{B}$ and $\mathrm{C}$ PHLF, no significant differences were demonstrated between AUCs of the CRMP index (0.850; 95\% CI, 0.733 to 0.967), MRE-LS ( $0.831 ; 95 \%$ CI, 0.718 to 0.944$)$, and ICG R15 (0.722; 95\% CI, 0.567 to 0.877) (Fig. 4E and F, Supplementary Table 3).

\section{DISCUSSION}

The predictive role of MRE-LS for PHLF was explored in HCC patients who underwent hepatic resection in this study. High preoperative MRE-LS was identified as a potent risk factor for PHLF, alongside traditional clini$\mathrm{cal} / \mathrm{biochemical}$ parameters such as low serum albumin level, high ALBI score, high serum AFP level, and major hepatic resection. High MRE-LS was also an independent prognostic marker for LSS in HCC patients who underwent surgical resection. Furthermore, we developed the CRMP index, a comprehensive clinical risk model for PHLF, by combining MRE-LS and other significant clini$\mathrm{cal} /$ biochemical variables. The CRMP index demonstrated excellent performance for predicting PHLF, particularly for grade B and C PHLF. As far as we know, this is the first 
study that developed an MRE-LS-based comprehensive clinical risk model for PHLF in HCC patients who underwent hepatic resection.

The incidence of PHLF has been reported as 5\% to 15\% in chronic liver disease patients who underwent hepatic resection. ${ }^{9,10}$ Similarly, in the present study, 24 patients (15\%) developed PHLF, among whom five (3.1\%) had only a minor, temporary deterioration in liver function that did not require intensive care, while $19(11.9 \%)$ had grade $\mathrm{B}$ or C PHLF urging invasive treatment or intensive care. PHLF is considered a feared complication as it is directly associated with poor prognosis in HCC patients following surgical resection. For patients with grade B and C PHLF in particular, peri-operative mortality has been reported as $12 \%$ and $54 \%$, respectively. ${ }^{37}$ Moreover, patients with PHLF showed markedly poorer survival than those without PHLF in our study. Among the eight mortalities that occurred in PHLF patients, seven occurred in those with grade $\mathrm{B}$ and C PHLF. Therefore, precise preoperative risk assessment for PHLF, particularly grade B and C PHLF, is essential for determining optimum treatment strategies and the improving prognosis of HCC patients.

Among the various biomarkers, MRE-LS showed the most potent discriminant power for predicting PHLF, followed by MELD score, ALBI score, and ICG R15. Especially in the major resection subgroup, MRE-LS showed excellent performance, which was sufficient to be used as a single independent biomarker (AUC, 0.843). ALBI score has previously been reported as a strong predictor of PHLF $^{21,38}$ and was revealed as a significant predictor of PHLF in the present study; it was thus incorporated into the CRMP index. However, ALBI score was insufficient to be used as a single biomarker for PHLF (AUC, 0.628). MELD score has also been reported as a potential prognostic factor in patients with liver cirrhosis, and ICG R15 is a widely used serum biomarker for evaluating preoperative hepatic reservoir. As such, both parameters were considered as potential predictors of PHLF; however, several recent studies reported insufficient performance of MELD score and ICG R15 as predictors of PHLF, ${ }^{39,40}$ and both MELD score and ICG R15 were not significantly associated with PHLF also in our study.

Prior to our study, there was only one other study which analyzed MRE-LS as a predictive marker for PHLF in HCC patients. Lee et al. ${ }^{17}$ reported that MRE-LS was an independent predictor of PHLF and a prognostic factor for overall survival in HCC patients. Our results corroborated with such findings, and in addition to this, we derived a comprehensive clinical risk model, named the CRMP index, as a more precise assessment of PHLF. The CRMP index consisted of five variables, including MRE-LS, serum albumin, serum AFP, major hepatic resection, and ALBI score. Besides MRE-LS, all of the included variables represent well-known traditional prognostic indicators of HCC. Performance of the CRMP index for predicting PHLF was excellent, with AUC of 0.877 in all included patients and of 0.923 for grade B and C PHLF in particular. In LOOCV, the AUC of CRMP score was 0.807 for predicting PHLF and 0.871 for predicting grade $\mathrm{B}$ and C PHLF. The CRMP index also showed outstanding performance compared to other single biomarkers in the subgroup analyses. Particularly, performance of the CRMP index for predicting grade B and C PHLF in patients who underwent minor hepatic resection was outstanding (AUC, 0.953). Such findings indicate that even in patients planning for minor hepatic resection, those with CRMP index $>-1.6$ should be monitored carefully due to a $100 \%$ sensitivity and $87 \%$ specificity chance of developing severe PHLF. Patient with higher CRMP index should be monitored more carefully, and if possible, be considered for alternative treatment modalities, such as transarterial chemoembolization or radiofrequency ablation. The CRMP index was also found to strongly associate with survival of HCC patients. Preoperative CRMP index could hence be used as not only a predictor of PHLF, but also a prognostic biomarker for survival in HCC patients.

The CRMP index is a useful and accurate risk score mainly based on MRE-LS. Although several prior researches have reported that TE-assessed LS (TE-LS) would be a promising predictor of PHLF, ${ }^{13,41,42}$ the performance of CRMP index or MRE-LS could not be directly compared with the TE-LS in the present study, because the values of preoperative TE-LS were not available in the majority of the included patients. However, as the MRE-LS has demonstrated excellent performance and reproducibility for assessing hepatic fibrosis compared to the TE-LS in many prior studies, ${ }^{43,44}$ we could carefully predict that MRL-LS might be superior to, or at least not inferior to, TE-LS also in predicting PHLF. Liver MRI is an essential modality for diagnosing HCC, and MRE can be easily incorporated into routine MRI protocols. Effective use of MRE-LS in clinical practice would lead to improved prognosis of HCC patients by facilitating the development of precision medicine.

There are several limitations in this study. First, no external validation was performed. Although LOOCV was performed and verified the excellent performance of the CRMP index, further external verification is required to apply it to real clinical practice. Second, FRV values were only available for a subset of patients. FRV is known to be one of the most important modifiable variable in predicting PHLF and postoperative mortality, but it was 
not significant in the present study. It might be caused by insufficient number of patients with available FRV values. Further research in developing a clinical risk model for PHLF using both FRV and MRE-LS in a larger cohort will lead to the development of a more improved model.

In conclusion, in our novel MRE-LS-based risk prediction model, the CRMP index provided excellent preoperative prediction for PHLF, particularly grade B and C PHLF, and also for survival in HCC patients. This risk prediction model carries great potential as a reference in the clinical decision-making process of HCC patients. External validation in large patient cohorts would be required.

\section{CONFLICTS OF INTEREST}

No potential conflict of interest relevant to this article was reported.

\section{ACKNOWLEDGEMENTS}

This research was funded by the Bio and Medical Technology Development Program of the National Research Foundation (NRF) funded by the Korean government (MSIT) (NRF- 2018M3A9E8023861).

\section{AUTHOR CONTRIBUTIONS}

Study concept and design: H.J.C., B.K., J.Y.C. Data acquisition: Y.H.A., M.S.S. Data analysis and interpretation: H.J.C., Y.H.A. Statistical analysis: J.W.E. Obtained funding: H.J.C. Material support: J.H., J.H.L., J.K.K. Study supervision: B.W.K., S.S.K. Statistical supervisor: B.L. Drafting of the manuscript: H.J.C., Y.H.A., B.K. Critical revision of the manuscript for important intellectual content: J.Y.C. All authors read and approved the final manuscript.

\section{ORCID}

Hyo Jung Cho https://orcid.org/0000-0003-4792-8335 Young Hwan Ahn https://orcid.org/0000-0001-9920-1846 Min Suh Sim https://orcid.org/0000-0003-3357-0803 Jung Woo Eun https://orcid.org/0000-0002-2461-6702 Soon Sun Kim https://orcid.org/0000-0002-6862-1896 Bong Wan Kim https://orcid.org/0000-0001-9059-0451 Jimi Huh https://orcid.org/0000-0002-8832-6165 Jei Hee Lee https://orcid.org/0000-0002-2231-820X Jai Keun Kim https://orcid.org/0000-0003-0767-6655
Buil Lee

https://orcid.org/0000-0001-9579-6280

Jae Youn Cheong https://orcid.org/0000-0001-6246-1783

Bohyun Kim

https://orcid.org/0000-0003-1157-415X

\section{SUPPLEMENTARY MATERIALS}

Supplementary materials can be accessed at https://doi. org/10.5009/gnl210130.

\section{REFERENCES}

1. European Association for the Study of the Liver. EASL clinical practice guidelines: management of hepatocellular carcinoma. J Hepatol 2018;69:182-236.

2. Njei B, Rotman Y, Ditah I, Lim JK. Emerging trends in hepatocellular carcinoma incidence and mortality. Hepatology 2015;61:191-199.

3. Hanouneh IA, Alkhouri N, Singal AG. Hepatocellular carcinoma surveillance in the 21st century: saving lives or causing harm? Clin Mol Hepatol 2019;25:264-269.

4. Bruix J, Sherman M; American Association for the Study of Liver Diseases. Management of hepatocellular carcinoma: an update. Hepatology 2011;53:1020-1022.

5. Wu KT, Wang CC, Lu LG, et al. Hepatocellular carcinoma: clinical study of long-term survival and choice of treatment modalities. World J Gastroenterol 2013;19:3649-3657.

6. European Association for the Study of the Liver; European Organisation for Research and Treatment of Cancer. EASLEORTC clinical practice guidelines: management of hepatocellular carcinoma. J Hepatol 2012;56:908-943.

7. Mizuguchi T, Kawamoto M, Meguro M, et al. Laparoscopic hepatectomy: a systematic review, meta-analysis, and power analysis. Surg Today 2011;41:39-47.

8. Asiyanbola B, Chang D, Gleisner AL, et al. Operative mortality after hepatic resection: are literature-based rates broadly applicable? J Gastrointest Surg 2008;12:842-851.

9. Gilg S, Sandström P, Rizell M, et al. The impact of posthepatectomy liver failure on mortality: a population-based study. Scand J Gastroenterol 2018;53:1335-1339.

10. Filicori F, Keutgen XM, Zanello M, et al. Prognostic criteria for postoperative mortality in 170 patients undergoing major right hepatectomy. Hepatobiliary Pancreat Dis Int 2012;11:507-512.

11. Hobeika C, Fuks D, Cauchy F, et al. Impact of cirrhosis in patients undergoing laparoscopic liver resection in a nationwide multicentre survey. Br J Surg 2020;107:268-277.

12. Procopet B, Fischer P, Horhat A, et al. Good performance of liver stiffness measurement in the prediction of postoperative hepatic decompensation in patients with cirrhosis 
complicated with hepatocellular carcinoma. Med Ultrason 2018;20:272-277.

13. Rajakannu M, Cherqui D, Ciacio O, et al. Liver stiffness measurement by transient elastography predicts late posthepatectomy outcomes in patients undergoing resection for hepatocellular carcinoma. Surgery 2017;162:766-774.

14. Han H, Hu H, Xu YD, Wang WP, Ding H, Lu Q. Liver failure after hepatectomy: a risk assessment using the pre-hepatectomy shear wave elastography technique. Eur J Radiol 2017;86:234-240.

15. Park CC, Nguyen P, Hernandez C, et al. Magnetic resonance elastography vs transient elastography in detection of fibrosis and noninvasive measurement of steatosis in patients with biopsy-proven nonalcoholic fatty liver disease. Gastroenterology 2017;152:598-607.

16. Imajo K, Kessoku T, Honda Y, et al. Magnetic resonance imaging more accurately classifies steatosis and fibrosis in patients with nonalcoholic fatty liver disease than transient elastography. Gastroenterology 2016;150:626-637.

17. Lee DH, Lee JM, Yi NJ, et al. Hepatic stiffness measurement by using MR elastography: prognostic values after hepatic resection for hepatocellular carcinoma. Eur Radiol 2017;27:1713-1721.

18. Sato N, Kenjo A, Kimura T, et al. Prediction of major complications after hepatectomy using liver stiffness values determined by magnetic resonance elastography. Br J Surg 2018;105:1192-1199.

19. Shi JY, Sun LY, Quan B, et al. A novel online calculator based on noninvasive markers (ALBI and APRI) for predicting post-hepatectomy liver failure in patients with hepatocellular carcinoma. Clin Res Hepatol Gastroenterol 2021;45:101534.

20. Fagenson AM, Gleeson EM, Karhadkar S, Di Carlo A, Pitt HA, Lau KN. Comparison of albumin-bilirubin and model for end-stage liver disease in predicting post-hepatectomy liver failure. J Am Coll Surg 2019;229:S170-S171.

21. Andreatos N, Amini N, Gani F, et al. Albumin-Bilirubin Score: predicting short-term outcomes including bile leak and post-hepatectomy liver failure following hepatic resection. J Gastrointest Surg 2017;21:238-248.

22. Toyoda H, Lai PB, O'Beirne J, et al. Long-term impact of liver function on curative therapy for hepatocellular carcinoma: application of the ALBI grade. Br J Cancer 2016;114:744750.

23. Lei JW, Ji XY, Hong JF, et al. Prediction of posthepatectomy liver failure using transient elastography in patients with hepatitis B related hepatocellular carcinoma. BMC Gastroenterol 2017;17:171.

24. Nishio T, Taura K, Koyama Y, et al. Prediction of posthepatectomy liver failure based on liver stiffness measurement in patients with hepatocellular carcinoma. Surgery 2016;159:399-408.
25. Xue LY, Fu TT, Ding H, et al. Predictive value of two-dimentional shear wave elastography in posthepatoectomy liver failure. Zhonghua Yi Xue Za Zhi 2020;100:3075-3080.

26. Cho HJ, Kim B, Kim HJ, et al. Liver stiffness measured by MR elastography is a predictor of early HCC recurrence after treatment. Eur Radiol 2020;30:4182-4192.

27. MR Elastography Biomarker Committee. QIBA profile: magnetic resonance elastography of the liver [Internet]. c2018 [cited 2020 Mar 30]. Available from: https://qibawiki. rsna.org/images/a/a5/MRE-QIBAProfile-2018-05-02-CONSENSUS.pdf.

28. Venkatesh SK, Yin M, Glockner JF, et al. MR elastography of liver tumors: preliminary results. AJR Am J Roentgenol 2008;190:1534-1540.

29. Marrero JA, Kulik LM, Sirlin CB, et al. Diagnosis, staging, and management of hepatocellular carcinoma: 2018 practice guidance by the American Association for the Study of Liver Diseases. Hepatology 2018;68:723-750.

30. Kim SY. Diagnosis of hepatocellular carcinoma: which MRI contrast agent? Which diagnostic criteria? Clin Mol Hepatol 2020;26:309-311.

31. Hwang DW, Han HS, Yoon YS, et al. Laparoscopic major liver resection in Korea: a multicenter study. J Hepatobiliary Pancreat Sci 2013;20:125-130.

32. Reddy SK, Pawlik TM, Zorzi D, et al. Simultaneous resections of colorectal cancer and synchronous liver metastases: a multi-institutional analysis. Ann Surg Oncol 2007;14:34813491.

33. Rahbari NN, Garden OJ, Padbury R, et al. Posthepatectomy liver failure: a definition and grading by the International Study Group of Liver Surgery (ISGLS). Surgery 2011;149:713-724.

34. Nousbaum JB, Cadranel JF, Bonnemaison G, et al. Clinical practice guidelines on the use of liver biopsy. Gastroenterol Clin Biol 2002;26:848-878.

35. Johnson PJ, Berhane S, Kagebayashi C, et al. Assessment of liver function in patients with hepatocellular carcinoma: a new evidence-based approach-the ALBI grade. J Clin Oncol 2015;33:550-558.

36. Clavien PA, Barkun J, de Oliveira ML, et al. The ClavienDindo classification of surgical complications: five-year experience. Ann Surg 2009;250:187-196.

37. Reissfelder C, Rahbari NN, Koch M, et al. Postoperative course and clinical significance of biochemical blood tests following hepatic resection. Br J Surg 2011;98:836-844.

38. Zhang ZQ, Xiong L, Zhou JJ, et al. Ability of the ALBI grade to predict posthepatectomy liver failure and long-term survival after liver resection for different BCLC stages of HCC. World J Surg Oncol 2018;16:208.

39. Yokoyama Y, Ebata T, Igami T, et al. The predictive value of indocyanine green clearance in future liver remnant for 
posthepatectomy liver failure following hepatectomy with extrahepatic bile duct resection. World J Surg 2016;40:14401447.

40. Alghamdi T, Abdel-Fattah M, Zautner A, Lorf T. Preoperative model for end-stage liver disease score as a predictor for posthemihepatectomy complications. Eur J Gastroenterol Hepatol 2014;26:668-675.

41. Kim SU, Ahn SH, Park JY, et al. Prediction of postoperative hepatic insufficiency by liver stiffness measurement (FibroScan $((\mathrm{R}))$ ) before curative resection of hepatocellular carcinoma: a pilot study. Hepatol Int 2008;2:471-477.

42. Cescon M, Colecchia A, Cucchetti A, et al. Value of transient elastography measured with FibroScan in predicting the outcome of hepatic resection for hepatocellular carcinoma. Ann Surg 2012;256:706-712.

43. Idilman IS, Li J, Yin M, Venkatesh SK. MR elastography of liver: current status and future perspectives. Abdom Radiol (NY) 2020;45:3444-3462.

44. Lee Yj, Lee JM, Lee JE, et al. MR elastography for noninvasive assessment of hepatic fibrosis: reproducibility of the examination and reproducibility and repeatability of the liver stiffness value measurement. J Magn Reson Imaging 2014;39:326-331. 